\title{
Nanoscale Structural Dynamics Probed by Coherent Ultrafast TEM
}

\author{
Armin Feist ${ }^{1 *}$, Thomas Danz ${ }^{1}$, Nara Rubiano da Silva ${ }^{1}$, Simon Vogelgesang ${ }^{1}$, Nora Bach ${ }^{1,2}$, \\ Till Domröse ${ }^{1}$, Sascha Schäfer ${ }^{1,2}$ and Claus Ropers ${ }^{1}$ \\ 1. 4th Physical Institute - Solids and Nanostructures, University of Göttingen, Göttingen, Germany. \\ 2. Institute of Physics, University of Oldenburg, Oldenburg, Germany. \\ * Corresponding author: armin.feist@uni-goettingen.de
}

Electron microscopy is tremendously successful in unravelling material structures and compositions on the atomic scale, with a temporal resolution governed by detector response times. Utilizing a stroboscopic approach, ultrafast electron diffraction [1] and microscopy [2] techniques provide for a unique access to processes on ultrashort time scales. In ultrafast transmission electron microscopy (UTEM), combining nanoscale spatial resolution of electron microscopy with femtosecond temporal resolution of optical spectroscopy, a pulsed electron beam of sub-picosecond duration is able to probe rapid processes. Drastically enhancing the achievable beam brightness in UTEM, the recent implementation of laser-triggered field emitters enables an advancement of the UTEM methodology towards coherent electron beam techniques [3].

Here, we describe the application of highly coherent electron pulses in UTEM for the probing of nanoscale dynamics in layered 2D materials by implementing ultrafast scanning convergent beam electron diffraction and ultrafast dark-field imaging.

The Göttingen UTEM instrument is based on a JEOL 2100F Schottky field emission TEM, which we modified to allow for optical sample excitation and the generation of photoelectron beams from nanoscale field emitters (cf. Fig. 1) [3,4]. Coherent ultrashort electron pulses with a normalized emittance of a few nm.mrad are achieved. Electron focal spot sizes down to $0.8 \mathrm{~nm}$, an electron pulse width of 200 fs (full-width-at-half-maximum) and a spectral bandwidth of $0.6 \mathrm{eV}$ are demonstrated [3]. In this contribution, the electron pulses of excellent spatial properties are utilized for two original applications.

At first, we show scanning ultrafast convergent beam electron diffraction (U-CBED) near the edge of an inhomogeneously excited single crystalline graphite thin film. The spatio-temporal local strain evolution is quantitatively analyzed with nanometer spatial and femtosecond temporal resolution (Fig. 2a-c) [5], complementing the information available in ultrafast bright-field imaging [6].

Furthermore, we demonstrate dark-field imaging of the charge-density wave phase transition in the correlated material $1 T-\mathrm{TaS}_{2}$, using either static imaging combined with in-situ laser excitation or with femtosecond resolution using a pulsed electron beam (Fig. 2d-f). Specifically, we track the evolution of domain patterns on femtosecond to picosecond time and nanometer length scales, extracting characteristic observables not accessible by ultrafast electron or x-ray diffraction [7].

In conclusion, we reported on the application of coherent ultrafast TEM for the study of nanoscale dynamics in layered 2D materials.

References: 
[1] RJD Miller, Science 343 (2014), p. 1108.

[2] AH Zewail, Science 328 (2010), p. 187.

[3] A Feist et al., Ultramicroscopy 176 (2017), p. 63.

[4] A Feist et al., Nature 521 (2015), p. 200.

[5] A Feist et al., Struct. Dyn. 5 (2018), p. 14302.

[6] DR Cremons et al., Nat. Commun. 7 (2016), p. 11230.

[7] T Danz et al., (in preparation).
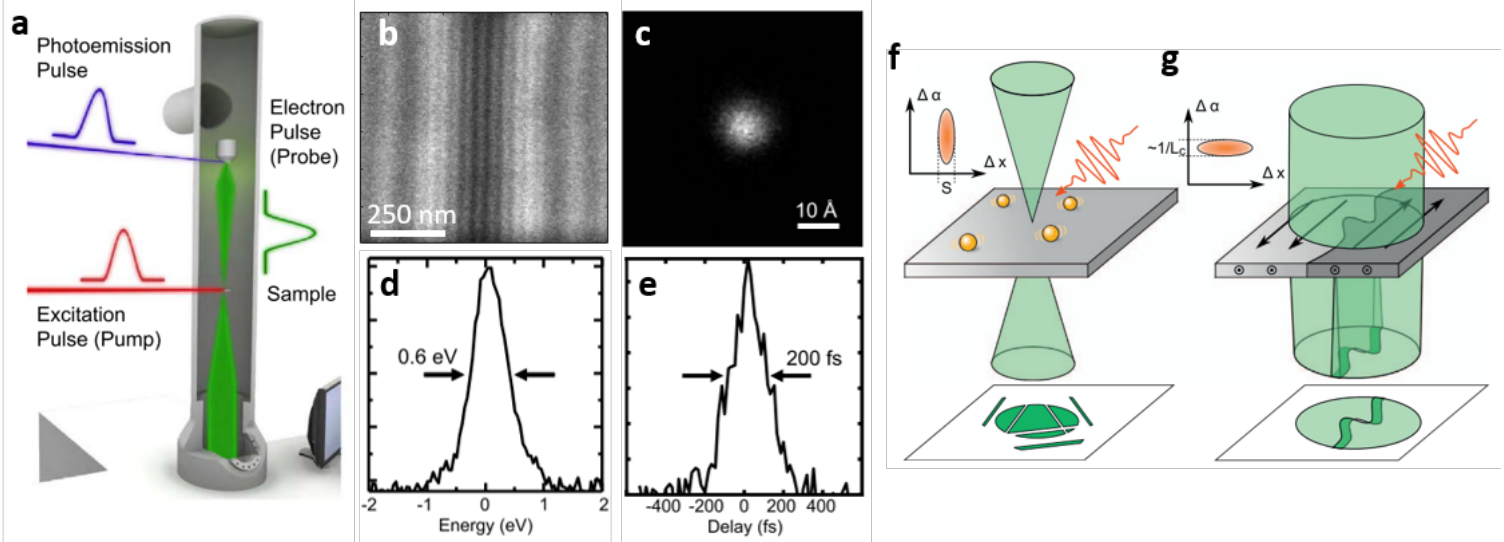

Figure 1. (a) Experimental scheme of UTEM: Ultrashort electron pulses, generated by nanotip photoemission (b), are accelerated and focused onto a sample (beam properties: (c)-(e)). The hereby generated low emittance electron pulses are ideally suited for (f) ultrafast local probing of nm-sized sample areas or (g) phase-contrast and dark-field imaging with $\mu \mathrm{m}$-scale coherence length. ((a)-(g) adapted from Ref. [3])
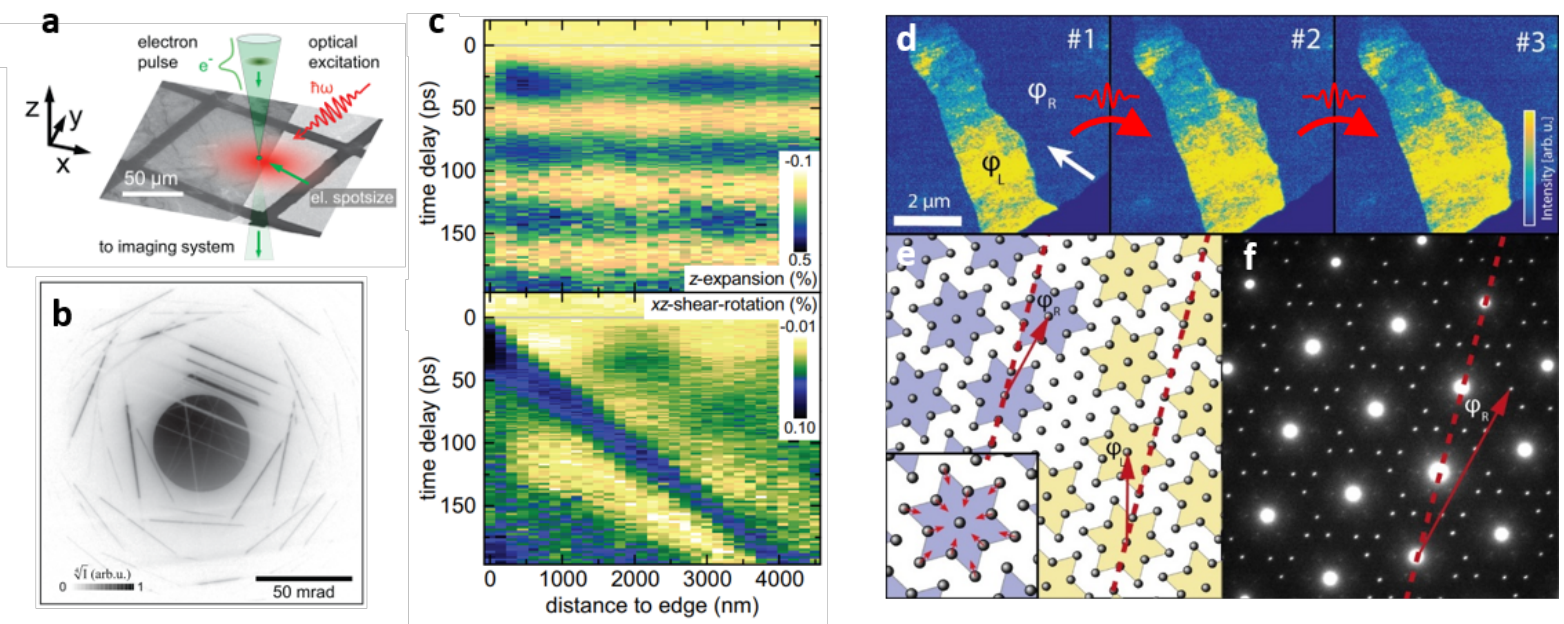

Figure 2. Probing of nanoscale structural dynamics in UTEM. (a) A laser-excited single-crystalline graphite thin film is probed by (b) ultrafast convergent beam electron diffraction (U-CBED). (c) Scanning U-CBED reveals the spatiotemporal distortion of the graphite membrane perpendicular to its edge. (d) Images of a thin $1 T-\mathrm{TaS}_{2}$ membrane at room temperature after structural transition between the two symmetric states of the nearly commensurate charge-density wave, induced by a series of singleshot laser pulses. (e) The specific shape of domains exhibiting different orientations is visualized in dark-field imaging by (f) filtering the associated diffraction peaks. ((a)-(c) adapted from Ref. [5]) 